\title{
Steady Synchronous Response and Balancing of Rotor Systems with Residual Shaft Bow
}

\author{
Harold D. Nelson \\ Mechanical and Aerospace Engineering, Arizona State University, Tempe, Arizona, USA
}

A procedure is presented for balancing a multi-degree-offreedom flexible rotor system including the effects of residual bow. The procedure is a generalization of methods presented by J. C. Nicholas, E. J. Gunter, and P. E. Allaire (1976a, 1976b) for a Laval-Jeffcott type rotor. These methods include: 1) treatment of the residual bow and unbalance as an equivalent unbalance force at the balance speed, 2) subtraction of the runout (residual bow response at near-zero speed) from the measured response at the balance speed, and 3) reducing the shaft response at the balance speed using data from a different measurement speed. The theoretical process is developed and an example, using simulated experimental data, is presented to illustrate the application of the three different strategies of balancing. The application of the balancing procedure requires the knowledge of a validated linear mathematical model for the rotor system. The mathematical model for the system must either be developed from theory, with appropriate experimental validation, or established directly from experimental data. Thus, application of the method is limited to those systems where mathematical models can be readily obtained.

Keywords Rotordynamics; Balancing; Residual bow

The steady dynamic response of high-speed rotating machinery is affected by many excitation sources including residual shaft bow and residual mass unbalance. In this work, the effects of residual shaft bow and residual mass unbalance on the steady state response of rotor systems are investigated and methods are suggested for balancing these types of systems. The suggested balancing procedures are a generalization of work originally

Received 20 October 2000; accepted 1 May 2001.

Address correspondence to Harold D. Nelson, Ph.D., Mechanical and Aerospace Engineering, Arizona State University, Box 876106, Tempe, AZ 85287-6106. presented by Nicholas, Gunter, and Allaire (1976a, 1976b) for a single-disk Laval-Jeffcott rotor system.

The rotor systems considered in this study include multishaft, multi-degree-of-freedom systems that are modeled with linear mechanisms. The support mechanisms are considered to be isotropic in this presentation, so all undamped rotor whirl modes and steady response orbits are circular. If the system possesses multiple shafts, residual bow and unbalance excitation exists for each shaft with a frequency of excitation coincident with the rotational speed of the associated shaft. In these situations, the response of the rotor system may be evaluated separately for the excitations associated with each shaft. The total system response, for a set of shaft excitations at different frequencies, is then a superposition of these individual responses.

The balancing of a multi-shaft system requires that each shaft of the system be balanced separately. In the presentation below, it is assumed that excitation is present only for a single shaft. The generalization to balancing of multi-shaft systems then requires a sequential balancing of each shaft of the system at the appropriate spin speed.

\section{REFERENCE SYSTEMS}

The lateral motion of a rotor system, in this work, is defined relative to two different reference systems as shown in Figure 1. The $X, Y, Z$-reference $(\mathcal{J})$ is a fixed (inertial) reference with the $Z$-axis coincident with the bearing centerline of the rotating assembly. Bearing misalignment is not considered in this investigation. The $x, y, z$-reference $(\mathcal{S})$ is a rotating reference system which rotates about its $z$-axis at a rate coincident with the spin speed of the rotating assembly under investigation. The $z$ and $Z$-axes are coincident.

The lateral motion of the rotor system, relative to $\mathcal{J}$, is described by a set of finite element equations associated with a set of shaft stations located along the $z$-axis of the shaft. At a typical station, say $i$, the lateral shaft motion is defined by two lateral translations $U_{i}, V_{i}$ in the $X, Y$-directions and two righthand-rule rotations $A_{i}, B_{i}$ also about the $X, Y$-axes. Thus, each station possesses four degrees-of-freedom. It is convenient, for 


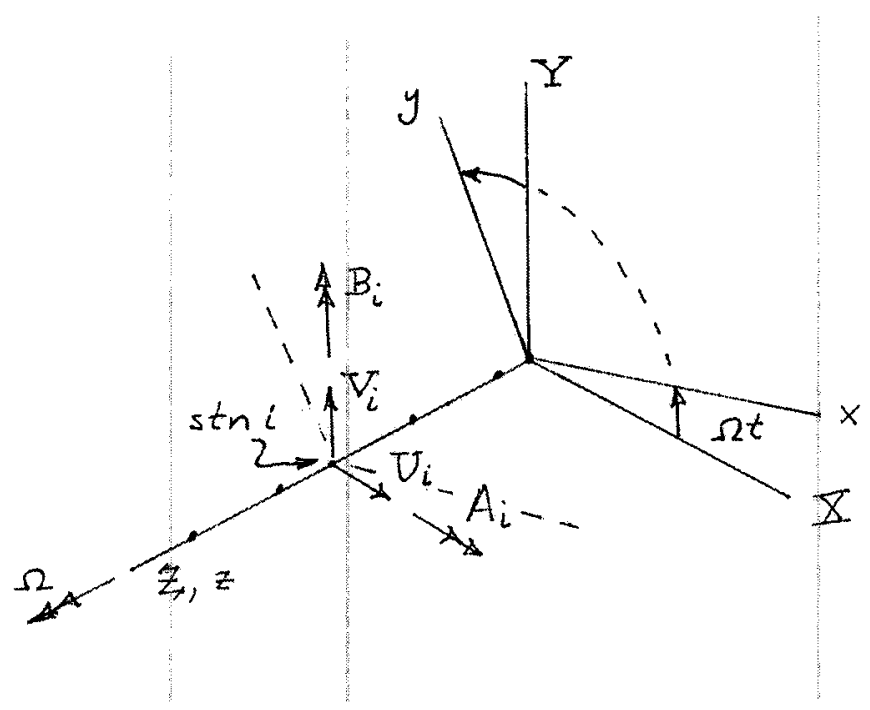

FIGURE 1

Reference systems and rotor displacements.

implementation of the balancing procedure presented here, to introduce the complex rotor displacements

$$
\begin{aligned}
W_{i} & =U_{i}+j V_{i} \\
\Gamma_{i} & =A_{i}+j B_{i}
\end{aligned}
$$

The finite element equations for modeling of rotordynamics systems, using complex coordinates, are presented by H. D. Nelson (1985) and others. Each finite element station possesses two complex degrees-of-freedom.

\section{SYSTEM EQUATIONS OF MOTION}

The equations of motion for a typical single-shaft rotor system, using complex coordinates, is of the general form:

$$
\begin{aligned}
& {[M]_{r a}\{\ddot{q}\}+\left([C]_{s}-\Omega[G]_{r a}\{\dot{q}\}+\left([K]_{s}+[K]_{r a}\right)\{q\}\right.} \\
& \quad=\left(\Omega^{2}\{Q\}_{u}+[K]_{r a}\{p\}_{r}\right) e^{j \Omega t}
\end{aligned}
$$

where the individual components of the equation of motion are defined in the nomenclature. This matrix equation is a set of complex, linear, second-order, ordinary differential equations. It possesses $2 n$ complex degrees-of-freedom where $n$ is the number of stations used in the finite element model. The residual bow vector $\{p\}_{r}$ consists of displacement components of the rotor relative to the rotating $x, y, z$-reference. Residual bow translational and rotational displacements at a typical station, $i$, are illustrated in Figure 2.

The residual bow displacement vector, $\{q\}_{r}$, relative to $\mathcal{J}$, is related to the residual bow displacement vector, $\{p\}_{r}$, by the

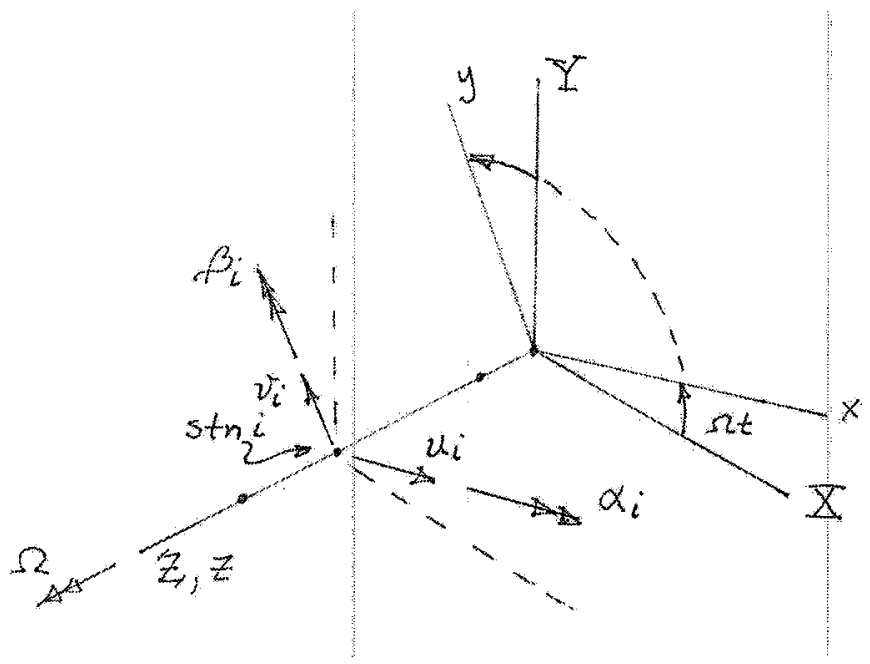

FIGURE 2

Residual bow displacements at a typical shaft station $i$.

rotation transformation

$$
\{q\}_{r}=\{p\}_{r} e^{j \Omega t} .
$$

\section{STEADY STATE RESPONSE} form

The steady state response, relative to $\mathcal{J}$, is of the general

$$
\{q\}=\{r(\Omega)\} e^{j \Omega t} .
$$

where $\{r(\Omega)\}$ is the response phasor of the system at the excitation frequency $\Omega$. Substitution of Equation (4) into the system equation of motion, Equation (2), yields the relation:

$$
[D(\Omega)]\{r(\Omega)\}=\Omega^{2}\{Q\}_{u}+[K]_{s}\{p\}_{r} .
$$

with:

$$
[D(\Omega)]=\left(\left([K]_{r a}+[K]_{s}\right)-\Omega^{2}[M]_{r a}\right)+i \Omega\left([C]_{s}-\Omega[G]_{r a}\right)
$$

defined here as the "dynamical matrix" of the rotor system. From Equation (5), the spin-speed dependent response phasor is given by the relation

$$
\begin{aligned}
\{r(\Omega)\} & =[H(\Omega)]_{u}\{Q\}_{u}+[H(\Omega)]_{r}\{p\}_{r} \\
& \left.=[D(\Omega)]^{-1}\left(\Omega^{2}\right)\{Q\}_{u}+[K]_{r a}\{p\}_{r}\right)
\end{aligned}
$$

with:

$$
\begin{aligned}
& {[H(\Omega)]_{u}=\Omega^{2}[D(\Omega)]^{-1} \quad \text { a) }} \\
& {[H(\Omega)]_{r}=[D(\Omega)]^{-1}[K]_{r a} \quad \text { b) }}
\end{aligned}
$$

defined respectively as the "unbalance influence coefficient matrix" and the "residual bow influence coefficient matrix" for the 
system. The excitation term, $\left(\Omega^{2}\{Q\}_{u}+[K]_{r a}\{p\}_{r}\right)$, on the right hand side of Equation (7) is a superposition of the spin speed squared unbalance excitation and the constant residual bow excitation.

\section{BALANCE METHODS: THEORY}

To implement the methods discussed below, the matrix of influence coefficients and the stiffness matrix for the rotating assembly must be known. In the discussion below, $\Omega_{b}$ is used to denote the rotor speed at which balancing is to occur, i.e., the balance speed.

\section{Method 1}

This balance method treats the superposition of the residual bow and unbalance excitations at the balance speed $\Omega_{b}$ as an "equivalent unbalance excitation." The desired result is for the response phasor of the system to be zero at the balance speed, $\Omega_{b}=\Omega_{m}$, as a result of adding a corrective unbalance vector $\{Q\}_{c 1}$ to the system. From Equation (7), setting the response phasor to zero, yields:

$$
\begin{aligned}
& \left\{r\left(\Omega_{b}\right)\right\}_{c 1}=\{0\}=\left[D\left(\Omega_{b}\right)\right]^{-1}\left(\Omega_{b}^{2}\{Q\}_{u}+[K]_{r a}\{p\}_{r}\right) \\
& +\left[H\left(\Omega_{b}\right)\right]_{u}\{Q\}_{c 1}=\left\{r\left(\Omega_{b}\right)\right\}_{o}+\left[H\left(\Omega_{b}\right)\right]_{u}\{Q\}_{c 1},
\end{aligned}
$$

where $\left\{r\left(\Omega_{b}\right)\right\}_{o}$ is the measured response of the system, at the balance speed $\Omega_{b}$, due to the original rotor state excitations. From Equation (9), the required unbalance correction vector for balance method 1 is

$$
\{Q\}_{c 1}=-[H(\Omega)]_{u}^{-1}\left\{r\left(\Omega_{b}\right)\right\}_{o}
$$

and the corrected response of the system, from Equation (7), is predicted to be

$$
\{r(\Omega)\}_{c 1}=[H(\Omega)]_{u}\left(\{Q\}_{u}+\{Q\}_{c 1}\right)+[H(\Omega)]_{r}\{p\}_{r} .
$$

\section{Method 2}

This method is identical with balance method 1 except the measured system runout (i.e., response as $\Omega \rightarrow 0$ ) is subtracted from the measured response phasor at the balance speed. In analogy with Equation (10), the required unbalance correction for balance method 2 is then

$$
\{Q\}_{c 2}=-[H(\Omega)]_{u}^{-1}\left(\left\{r\left(\Omega_{b}\right)\right\}_{o}-\{r(0)\}_{o}\right),
$$

and the corrected response of the system, from Equation (7), is predicted to be

$$
\{r(\Omega)\}_{c 2}=[H(\Omega)]_{u}\left(\{Q\}_{u}+\{Q\}_{c 2}\right)+[H(\Omega)]_{r}\{p\}_{r}
$$

\section{Method 3}

The purpose of balance method 3 is to utilize measured data at a measurement speed $\Omega_{m}$ to "balance" the rotor system at a different speed defined here as the balance speed, $\Omega_{b}$. Before balancing, the steady system response phasor, associated with the original rotor state, at the measurement speed $\Omega_{m}$, is

$$
\left\{r\left(\Omega_{m}\right)\right\}_{o}=\left[H\left(\Omega_{m}\right)\right]_{u}\{Q\}_{u}+\left[H\left(\Omega_{m}\right)\right]_{r}\{p\}_{r}
$$

The unbalance force vector, from Equation (14), is then

$$
\{Q\}_{u}=\left[H\left(\Omega_{m}\right)\right]_{u}^{-1}\left\{r\left(\Omega_{m}\right)\right\}_{o}-\left[H\left(\Omega_{m}\right)\right]_{u}^{-1}\left[H\left(\Omega_{m}\right)\right]_{r}\{p\}_{r} .
$$

After the system is balanced, the desired value for the system response phasor at the balance speed, $\Omega_{b}$, is zero. From Equation (7), including the correction unbalance vector,

$$
\left\{r\left(\Omega_{b}\right)\right\}_{c 3}=\{0\}=\left[H\left(\Omega_{b}\right)\right]_{u}\left(\{Q\}_{u}+\{Q\}_{c 3}\right)+\left[H\left(\Omega_{b}\right)\right]_{r}\{p\}_{r} .
$$

From Equation (16), using the identity $[H(\Omega)]_{u}^{-1}[H(\Omega)]_{r}=$ $\frac{1}{\Omega^{2}}[K]_{r a}$ and Equation (15), the corrective unbalance vector for balance method 3 is

$$
\{Q\}_{c 3}=-\left[H\left(\Omega_{m}\right)\right]_{u}^{-1}\left\{r\left(\Omega_{m}\right)\right\}_{o}+\frac{1}{\Omega_{b}^{2}} \frac{\Omega_{b}^{2}-\Omega_{m}^{2}}{\Omega_{m}^{2}}[K]_{r a}\{p\}_{r}
$$

and the corrected response of the system, from Equation (7), is predicted to be

$$
\{r(\Omega)\}_{c 3}=[H(\Omega)]_{u}\left(\{Q\}_{u}+\{Q\}_{c 3}\right)+[H(\Omega)]_{r}\{p\}_{r} .
$$

\section{BALANCING METHODS: IMPLEMENTATION}

Implementation of the balancing methods, described above, requires measurement of the steady response phasor, $\{r(\Omega)\}_{o}$, and the residual bow (runout) vector $\{p\}_{r}$. Each of these complex vectors is of order $2 n$ and includes a complex translation, Equation (1(a)), and complex rotation Equation (1(b)), at each finite element station of the shaft. It is generally not practical and usually impossible to make $2 n$ separate measurements on a typical rotor system. In addition, the measurement of rotation displacements is not normally a feasible task. In the unlikely event that one could actually make these $2 n$ measurements, the resulting unbalance correction vector would then need to include both mass unbalance and mass-moment unbalance at each station. The addition of such a large number of unbalance corrections to a rotor system is clearly unrealistic.

In order to render the above balancing procedures feasible, it is necessary to introduce a coordinate reduction strategy to reduce the order of the system model to a tractable level. In addition, it is probably advisable to retain only translational 
coordinates so the resulting unbalance correction vector only includes unbalance forces and no unbalance moments. The method of static condensation is presented here as a mechanism for coordinate reduction. To illustrate the reduction process, consider the five-station (10 complex degrees-of-freedom-a complex translation and complex rotation at each station) example rotor system shown in Figure 3. The coordinate vector that defines the motion of this system is:

$$
\{q\}=\left\lfloor\begin{array}{llllllllll}
W_{1} & \Gamma_{1} & W_{2} & \Gamma_{2} & W_{3} & \Gamma_{3} & W_{4} & \Gamma_{4} & W_{5} & \Gamma_{5}
\end{array}\right\rfloor^{T} .
$$

For purposes of this example, retain only the translational coordinates $\left(W_{2}, W_{3}, W_{4}\right)$ and thereby reduce the complex degrees-offreedom of the system from ten to three. The first step in this process is to reorder the system coordinate vector, e.g., as follows:

$$
\{\widehat{q}\}=\left\lfloor\begin{array}{llllllllll}
W_{2} & W_{3} & W_{4} & W_{1} & \Gamma_{1} & \Gamma_{2} & \Gamma_{3} & W_{5} & \Gamma_{4} & \Gamma_{5}
\end{array}\right\rfloor^{T},
$$

using a reordering transformation of coordinates of the form:

$$
\{q\}=[R]\{\bar{q}\}
$$

Introduction of Equation (21) into the set of system equations, Equation (2), and pre-multiplication by the transpose of $[R]$, yields the system equations associated with a coordinate vector as defined by relation (20). These system equations have the same form as Equation (2), with the understanding that the arrays are reordered such that they are compatible with the new coordinate ordering.

The second step in the coordinate reduction process is to define the subset of rotor displacements $\{q\}_{a}=\left\lfloor\begin{array}{lll}W_{2} & W_{3} & W_{4}\end{array}\right\rfloor$ as active (master) coordinates and the remaining subset of rotor displacements, $\{q\}_{d}=\left\lfloor W_{1} \Gamma_{1} \Gamma_{2} \Gamma_{3} W_{5} \Gamma_{4} \Gamma_{5}\right\rfloor$, as dependent (slave) coordinates. A purely static relationship between the dis- placements and associated forces may then be defined in the form:

$$
\left[\begin{array}{cc}
{[K]_{a a}} & {[K]_{a d}} \\
{[K]_{d a}} & {[K]_{d d}}
\end{array}\right]\left\{\begin{array}{l}
\{q\}_{a} \\
\{q\}_{d}
\end{array}\right\}=\left\{\begin{array}{l}
\{Q\}_{a} \\
\{Q\}_{d}
\end{array}\right\}
$$

A relationship may be obtained between the dependent and active subsets of rotor coordinates by assuming that the static force vector associated with the dependent subset of coordinates is zero. Thus, from the second row of Equation (22):

$$
\{q\}_{d}=-[K]_{d d}^{-1}[K]_{d a}\{q\}_{a} .
$$

This relationship and an identity relation may be combined to yield the transformation of coordinates

$$
\{\widehat{q}\}=\left\{\begin{array}{l}
\{q\}_{a} \\
\{q\}_{d}
\end{array}\right\}=\left[\begin{array}{c}
{[I]} \\
-[K]_{d d}^{-1}[K]_{d a}
\end{array}\right]\{q\}_{a}=[S]\{q\}_{a} .
$$

Introduction of Equation (24) into a reordered set of system equations, Equation (2), and pre-multiplication by the transpose of $[S]$, yields the reduced-order set of system equations associated with the active set of coordinates $\{q\}_{a}$. These system equations are of same form as Equation (2), except the order has been reduced and the system arrays transformed appropriately. These equations are now in a form that may be tractable for implementation of the balance methods discussed above.

In the remainder of this presentation it is assumed that the system equations have been reduced to a tractable level using static condensation of some other coordinate reduction strategy. Clearly, an analyst must be vigilant when using coordinate reduction to be certain that the reduced order equations provide an acceptable level of accuracy in defining the dynamic characteristics of the system for the frequency range of interest. Additional discussion on the topic of coordinate reduction may be found in the work by J. J. Yu and A. Craggs (1995).

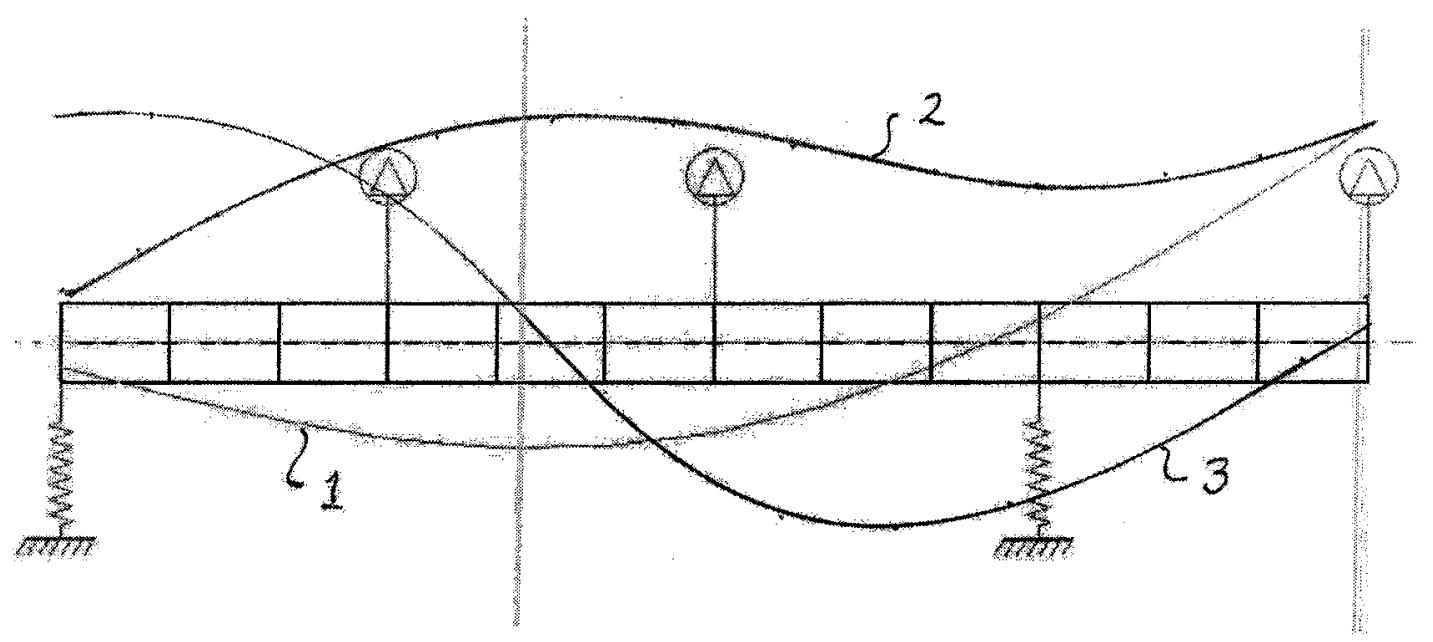

FIGURE 3

Example rotor system \& critical modes. 


\section{EXAMPLE}

The rotor system shown in Figure 3, is an example system to illustrate the application of the balancing strategies defined above. Experimental results for this example system are simulated using a rotordynamics analysis computer program. This program models the rotor system as an assemblage of rotating finite shaft elements with residual bow, unbalanced rigid disks, and linear bearings.

The rotor system is modeled with five stations interconnected by solid cross-section rotating shaft-elements each of length 12 inches $(304.8 \mathrm{~mm})$ and diameter three inches $(76.2 \mathrm{~mm})$. The modulus of elasticity and mass density of the shaft elements are $E=30.10^{6} \mathrm{lb}_{\mathrm{f}} / \mathrm{in}^{2}(206.8 \mathrm{GPa})$ and $\rho=0.286 \mathrm{lb} / \mathrm{in}^{3}$ $\left(7,916 \mathrm{~kg} / \mathrm{m}^{3}\right)$ respectively. Rigid disks with identical inertial properties $\left[m=0.12567 \mathrm{lb}_{\mathrm{f}^{-}} \mathrm{s}^{2} / \mathrm{in}(22.008 \mathrm{~kg}), I_{d}=1.131 \mathrm{lb}_{\mathrm{f}^{-}}\right.$ $\mathrm{s}^{2}$-in $\left(0.12779 \mathrm{~kg}-\mathrm{m}^{2}\right), I p=2.260\left(\mathrm{lb}_{\mathrm{f}}-\mathrm{s}^{2}\right.$-in $\left.\left(0.25557 \mathrm{~kg}-\mathrm{m}^{2}\right)\right]$ are present at stations 2,3 , and 5. Identical linear bearings with stiffness $k=250,000 \mathrm{lb}_{\mathrm{f}} / \mathrm{in}(43.78 \mathrm{MN} / \mathrm{m})$ and viscous damping $c=100 \mathrm{lb}_{\mathrm{f}}$-s/in $(17.51 \mathrm{KN}-\mathrm{s} / \mathrm{m})$ support the rotating assembly at stations 1 and 4.

For the purpose of simulating experimental data, the cg eccentricity of each rigid disk is selected as $e=0.002$ in $(50.8 \mu \mathrm{m})$ at an angle of $\beta=90$ degrees rotated positive from a reference line on the shaft which is parallel with the rotating $x$-axis. The rotating shaft elements are assumed to be balanced. The initial shaft bow is assumed to be confined to the $x, z$-plane for this example and is defined as a smooth curve through values of 0.889 mils $(22.57 \mu \mathrm{m})$ at stations 2 and 3 and a value of -1.778 mils $(-45.16 \mu \mathrm{m})$ at station 5 . The rotordynamics program uses a spline-fit to obtain the residual-bow displacements and rotations at each rotor station of the system.

A critical speed analysis of this system predicts forward undamped critical speeds at approximately $n_{1}=4,428 \mathrm{rpm}, n_{2}=$ $6,239 \mathrm{rpm}$ and $n_{3}=18,316 \mathrm{rpm}$. The synchronous mode shapes associated with these three critical speeds are shown in Figure 3.

Displacement measurements (amplitude and phase lag) are taken at stations 2, 3, and 5 at a measurement spin speed of $3,500 \mathrm{rpm}$. This speed is selected because it is below the first critical speed at a state where the phase lag of each of the displacements is relatively steady, i.e., small changes in the spin
TABLE I

Steady state response data

\begin{tabular}{|c|c|c|c|c|c|c|}
\hline \multirow[b]{3}{*}{ Stn no } & \multicolumn{3}{|c|}{$3,500 \mathrm{rpm}$} & \multicolumn{3}{|c|}{$0 \mathrm{rpm}$} \\
\hline & \multicolumn{2}{|c|}{ Amplitude } & \multirow{2}{*}{$\begin{array}{c}\text { Angle } \\
\text { deg }\end{array}$} & \multicolumn{2}{|c|}{ Amplitude } & \multirow{2}{*}{$\begin{array}{c}\text { Angle } \\
\text { deg }\end{array}$} \\
\hline & mils & $\mu \mathrm{m}$ & & mils & $\mu \mathrm{m}$ & \\
\hline 2 & 2.34 & 59.44 & 350 & 0.889 & 22.58 & 0 \\
\hline 3 & 2.3 & 58.42 & 348 & 0.889 & 22.58 & 0 \\
\hline 5 & 4.71 & 119.63 & 193 & 1.778 & 45.21 & 180 \\
\hline
\end{tabular}

speed do not significantly affect the phase lag measurements. The measured displacement amplitudes and phase lags at 3,500 and 0 (runout) rpm are listed in Table I.

The calculated unbalance correction vectors for balance methods 1, 2, and 3, using Equations (10, 12, and 17) respectively, are shown in Table II.

The unbalance corrections listed in Table II were applied to the rotor system and the steady state response was evaluated for each case. A Bode plot of the response at stations 2, 3, and 5 are graphed in Figure 4 for a) the original state and after applying balance corrections for: $\mathrm{b}$ ) balance method \#1, c) balance method \#2, d) balance method \#3 with $\Omega_{b}=4,428 \mathrm{rpm}$, and e) balance method \#3 with $\Omega_{b}=6,239 \mathrm{rpm}$.

The response amplitudes (inches) versus the rotor spin speed (rpm) are shown in Figure 4 for the original rotor state and for the four balance methods listed in Table II. The steady state response peak amplitudes associated with these graphs are listed in Table III.

\section{RESULTS}

The peak rotor responses at stations 2,3 , and 5 were reduced by approximately $50 \%$ at both critical speeds compared to the original state using the balance corrections from balance method 1. The measurement speed (and also the balance speed for this case) was $\Omega_{m}=\Omega_{b}=3,500 \mathrm{rpm}$. An inspection of the response (Figure $4(b)$ ) plot reveals that all three response amplitudes were reduced essentially to zero at this balance speed.

TABLE II

Calculated unbalance corrections

\begin{tabular}{|c|c|c|c|c|c|c|c|c|c|c|c|c|}
\hline \multirow[b]{4}{*}{ Stn no } & \multirow{2}{*}{\multicolumn{3}{|c|}{ Method 1}} & \multirow{2}{*}{\multicolumn{3}{|c|}{ Method 2}} & \multicolumn{3}{|c|}{ Method 3} & \multicolumn{3}{|c|}{ Method 3} \\
\hline & & & & & & & \multicolumn{3}{|c|}{$\Omega_{b}=4,428 \mathrm{rpm}$} & \multicolumn{3}{|c|}{$\Omega_{b}=6,239 \mathrm{rpm}$} \\
\hline & \multicolumn{2}{|c|}{ Amplitude } & \multirow{2}{*}{$\begin{array}{c}\text { Ph lag } \\
\text { deg }\end{array}$} & \multicolumn{2}{|c|}{ Amplitude } & \multirow{2}{*}{$\begin{array}{c}\text { Ph lag } \\
\text { deg }\end{array}$} & \multicolumn{2}{|c|}{ Amplitude } & \multirow{2}{*}{$\begin{array}{c}\text { Ph lag } \\
\text { deg }\end{array}$} & \multicolumn{2}{|c|}{ Amplitude } & \multirow{2}{*}{$\begin{array}{r}\text { Ph lag } \\
\text { deg }\end{array}$} \\
\hline & $\mathrm{lb}_{\mathrm{f}}-\mathrm{s}^{2}$ & $\mathrm{~kg}-\mathrm{mm}$ & & $1 b_{f}-s^{2}$ & $\mathrm{~kg}-\mathrm{mm}$ & & $\mathrm{lb}_{\mathrm{f}}-\mathrm{s}^{2}$ & $\mathrm{~kg}-\mathrm{mm}$ & & $\mathrm{lb}_{\mathrm{f}}-\mathrm{s}^{2}$ & $\mathrm{~kg}-\mathrm{mm}$ & \\
\hline 2 & $3.81 \mathrm{E}-04$ & 1.695 & 230 & $3.30 \mathrm{E}-04$ & 1.469 & 229 & $4.08 \mathrm{E}-04$ & 1.814 & 226 & 4.31E-04 & 1.919 & 223 \\
\hline 3 & $3.84 \mathrm{E}-04$ & 1.709 & 214 & $2.75 \mathrm{E}-04$ & 1.224 & 249 & $2.27 \mathrm{E}-04$ & 1.009 & 252 & $2.55 \mathrm{E}-04$ & 1.134 & 302 \\
\hline 5 & $3.81 \mathrm{E}-04$ & 1.696 & 323 & $3.11 \mathrm{E}-04$ & 1.383 & 311 & $2.91 \mathrm{E}-04$ & 1.297 & 308 & $2.42 \mathrm{E}-04$ & 1.076 & 288 \\
\hline
\end{tabular}



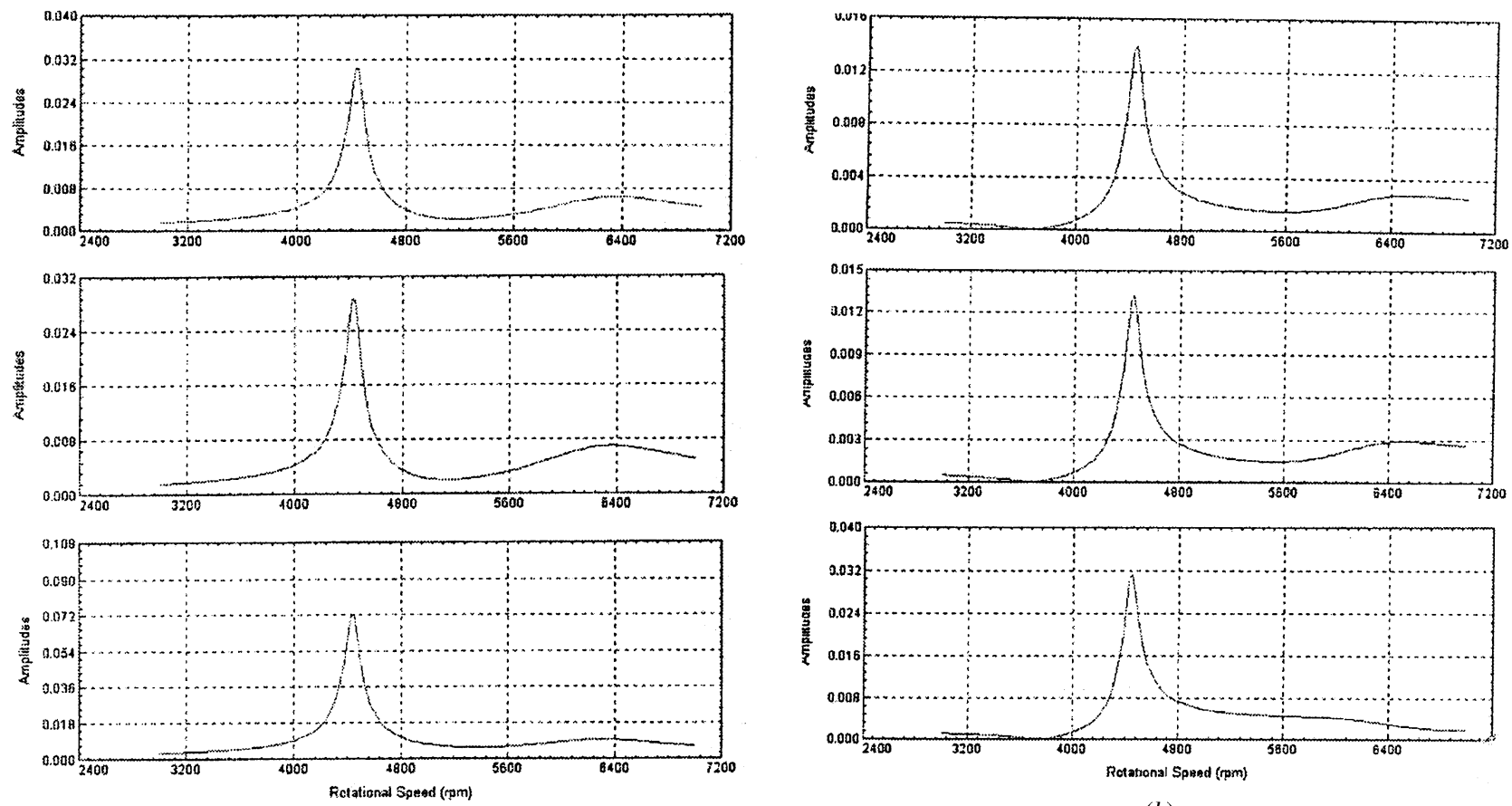

(a)

(b)
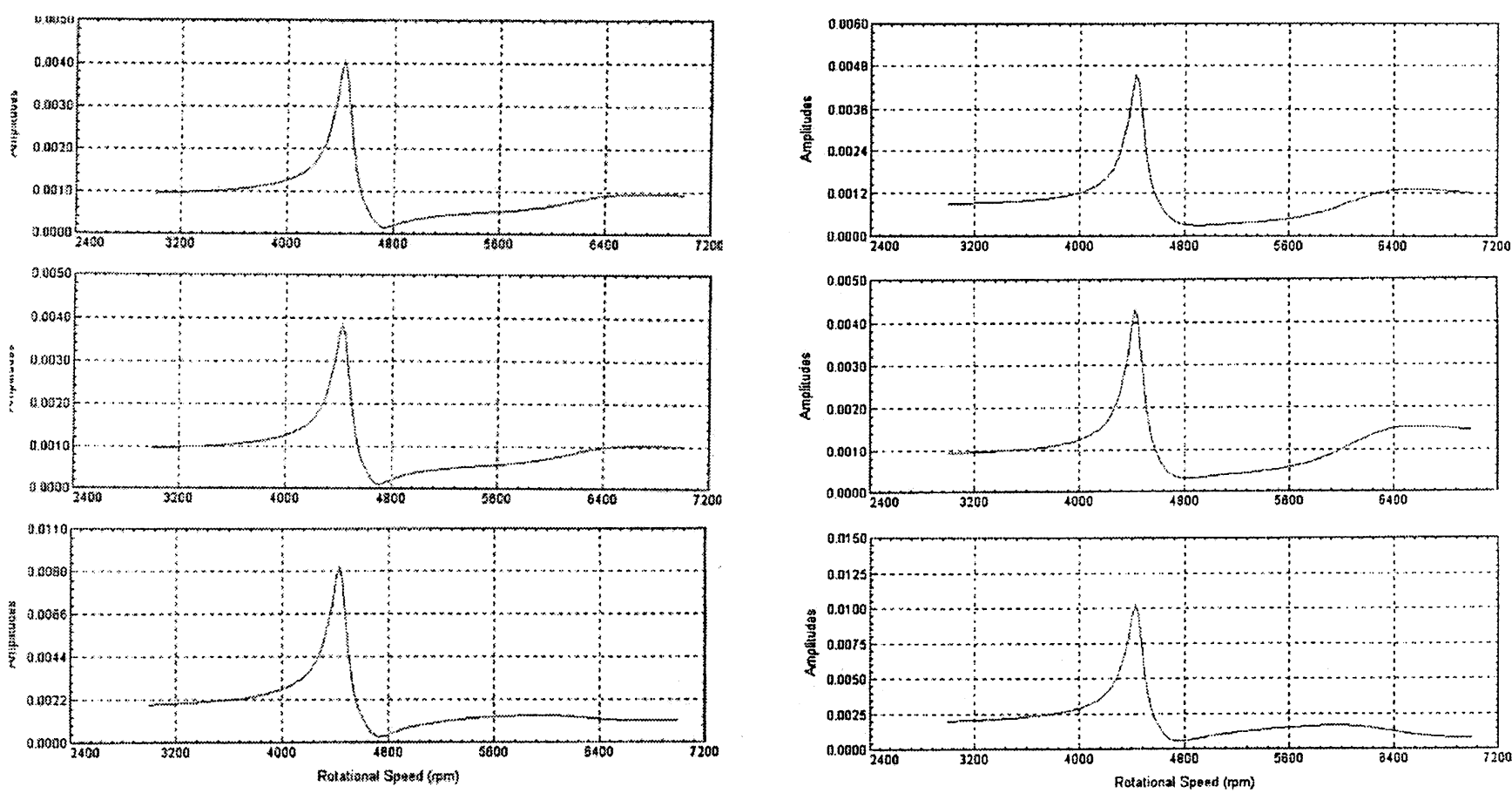

(c)

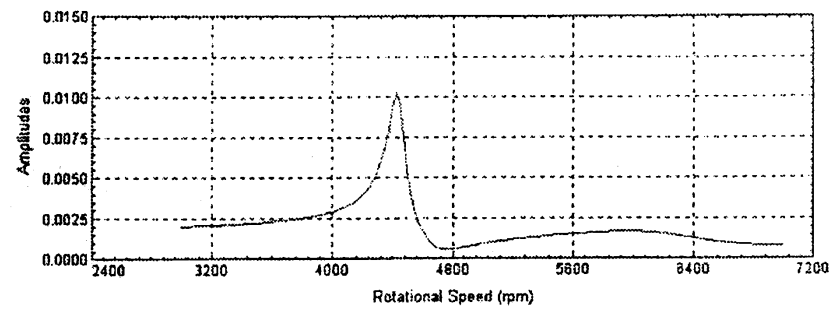

$(d)$

FIGURE 4

Steady state response: Before and after balancing. (a) Original state, $(b)$ after balance method 1, $(c)$ after balance method 2, (d) after balance method $3\left(\Omega_{b}=4,428 \mathrm{rpm}\right),(e)$ after balance method $3\left(\Omega_{b}=6,239 \mathrm{rpm}\right)$. 

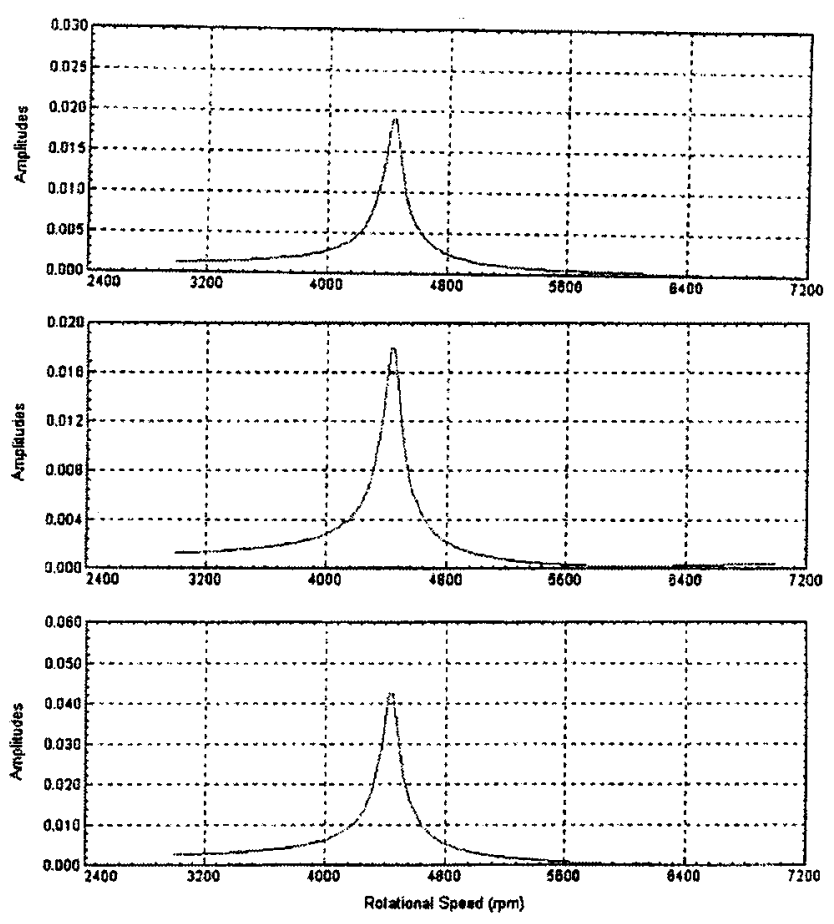

(e)

FIGURE 4

(Continued)

The application of balance corrections associated with balance method 2 (Figure $4(c)$ ) provided over $80 \%$ reduction in the peak amplitudes. The response amplitudes at the balance speed of $\Omega_{m}=\Omega_{b}=3,500 \mathrm{rpm}$ were slightly higher than the runout amplitudes at the response locations.

The application of balance corrections associated with balance method 3 (Figure $4(d)$ ) was first tested using a measurement speed of $\Omega_{m}=3,500 \mathrm{rpm}$ and a balance speed of $\Omega_{b}=4,428 \mathrm{rpm}$. The reduction in peak amplitudes was comparable to those experienced using balance method 2 . The theory for this method predicts that these amplitudes should be reduced to zero provided all measurements and models are perfect. Quite clearly, perfection does not exist in the reduced order model used to estimate the balance corrections. For instance, the first critical speed of the reduced order model is approximately 4,438 rpm, while the critical speed of the simulated system is $4,428 \mathrm{rpm}$. The sharpness of the response curves at the first critical speed is quite high. Thus, the balancing process is quite sensitive to the difference between the critical speed of the system mathematical model and the real system.

A second application of balance corrections associated with balance method 3 (Figure 4(e)) was tested using a measurement speed of $\Omega_{m}=3,500 \mathrm{rpm}$ and a balance speed of $\Omega_{b}=6,239 \mathrm{rpm}$. In this case, the amplitudes of all responses at the balance speed were reduced essentially to zero. The sharpness of the response curves at the second critical speed is significantly lower than at the first critical speed. Thus, the sensitivity to the difference in the predicted and real critical speed is much lower.

\section{CONCLUSIONS}

The general equations of motion are presented for a multidegree-of-freedom, multi-shaft rotor dynamic system modeled with linear finite elements and including excitation from residual shaft bow and residual unbalance. The lateral displacements of the rotor cross-section are defined in terms of complex translations and rotations at each station. Thus, the equations of motion are also written in complex form.

The solution for the steady state response phasor, as a function of the rotor spin speed, is developed including the contribution from the constant rotating force associated with residual bow and the spin speed squared rotating force associated with residual unbalance.

Three methods of balancing a rotor system are presented; based on the work of Nicholas, Gunter, and Allaire (1976a, 1976b); and these methods are applied to a five-station (10 cdof) sample rotor system. The results associated with these three methods and this example suggest that the proposed methods may be practical for some special situations. The methods require the knowledge of a mathematical model for the rotor system that accurately predicts real system behavior over the

TABLE III

Peak amplitude rotor response at critical speeds

\begin{tabular}{|c|c|c|c|c|c|c|c|c|c|c|}
\hline \multirow[b]{3}{*}{$\Omega$} & \multirow{2}{*}{\multicolumn{2}{|c|}{ Original state }} & \multirow{2}{*}{\multicolumn{2}{|c|}{$\frac{\text { Balance method } 1}{\Omega_{b}=3,500 \mathrm{rpm}}$}} & \multirow{2}{*}{\multicolumn{2}{|c|}{$\frac{\text { Balance method } 2}{\Omega_{b}=3,500 \mathrm{rpm}}$}} & \multirow{2}{*}{\multicolumn{2}{|c|}{$\frac{\text { Balance method } 3}{\Omega_{b}=4,428 \mathrm{rpm}}$}} & \multirow{2}{*}{\multicolumn{2}{|c|}{$\frac{\text { Balance method } 3}{\Omega_{b}=6,239 \mathrm{rpm}}$}} \\
\hline & & & & & & & & & & \\
\hline & 4,428 & 6,239 & 4,428 & 6,239 & 4,428 & 6,239 & 4,428 & 6,239 & 4,428 & 6,239 \\
\hline \multicolumn{11}{|c|}{ Stn. no. } \\
\hline 2 & 30 & 7 & 14 & 3 & 4 & 1 & 4 & 1.5 & 19 & 0 \\
\hline 3 & 28 & 7 & 13 & 3 & 4 & 1 & 4 & 1.5 & 18 & 0 \\
\hline 5 & 72 & 9 & 32 & 4 & 9 & 1 & 10 & 2 & 42 & 0 \\
\hline
\end{tabular}


frequency range of interest. This model then uses experimental data to estimate corrective unbalance vectors associated with each unbalance method. The corrective unbalance vector includes an unbalance force for each translation coordinate and an unbalance moment for each rotation coordinate included in the model. Thus, a practical model should probably only include a small number of translational coordinates so only unbalance forces are required to be applied to the system to provide an improvement in performance.

The requirements described above clearly provide very severe limitations for the use of the methods for balancing of practical rotor systems. For those special situations, however, when accurate mathematical models exist for a system, the methods may prove to be highly effective.

\section{NOMENCLATURE}

$\begin{array}{ll}\text { cdof } & \text { complex degrees-of-freedom } \\ {[C]} & \text { dissipation matrix } \\ {[D(\Omega)]} & \text { dynamical matrix } \\ \text { dof } & \text { degrees-of-freedom } \\ e & \text { cg eccentricity } \\ E & \text { modulus of elasticity } \\ \mathcal{J} & X, Y, Z \text { (fixed) reference } \\ {[G]} & \text { gyroscopic matrix } \\ {[H(\Omega)]} & \text { influence coefficient matrix } \\ I & \text { mass moment of inertia } \\ j & \text { imaginary element } \\ {[K]} & \text { stiffness matrix } \\ {[M]} & \text { inertia (mass) matrix } \\ n & \text { number of rotor stations } \\ \{p\} & \text { residual bow vector re: } \mathcal{S} \\ \{q\} & \text { displacement vector re: } \mathcal{J} \\ \{\dot{q}\} & \text { velocity vector re: } \mathcal{J} \\ \{\ddot{q}\} & \text { acceleration vector re: } \mathcal{J} \\ \{Q\} & \text { excitation force vector re: } \mathcal{J} \\ \{r(\Omega)\} & \text { displacement response phasor re: } \mathcal{J} \\ {[R]} & \text { reordering transformation matrix } \\ \mathcal{S} & x, y, z \text { (spin) reference } \\ {[S]} & \text { static condensation transf. matrix } \\ t & \text { time } \\ U, V & \text { lateral transl. in the } X, Y \text {-directions } \\ u, v & \text { res. bow. transl. in the } x, y \text {-dir. }\end{array}$

$W, \Gamma \quad$ complex translation, rotation re: $\mathcal{J}$

$w, \gamma \quad$ res. bow complex transl., rot. re: $\mathcal{S}$

\section{Greek Symbols}

$A, B \quad$ rotations about the $X, Y$-axes

$\alpha, \beta \quad$ res. bow rot. about the $x, y$-axes

$\beta \quad$ cg angle + ccw re: $x$-axis

$\rho \quad$ mass density

\section{Subscripts}

$\begin{array}{ll}a & \text { active (master) } \\ b & \text { balance speed } \\ c \mu & \text { bal. cor. for method } \mu(\mu=1,2,3) \\ d & \text { dependent (slave), diametral } \\ i & \text { rotor station index }(i=1, \ldots n) \\ m & \text { measurement speed } \\ n & \text { number of rotor stations } \\ o & \text { original rotor state } \\ p & \text { polar } \\ r & \text { residual bow } \\ r a & \text { rotating assembly } \\ s & \text { support mechanisms } \\ u & \text { unbalance }\end{array}$

\section{Overbars}

$-\quad$ reordered vector
$-\quad$ active set of coordinates, $\{q\}_{a}$

\section{REFERENCES}

Nelson, H. D. 1985. Rotor dynamics equations in complex form. Journal of Vibration, Acoustics, Stress and Reliability in Design, Trans. ASME 107(4) October: 460-461.

Nicholas, J. C., Gunter, E. J., and Allaire, P. E. 1976a. Effect of residual shaft bow on unbalance response and balancing of a single mass flexible rotor, Part 1: Unbalance response. Journal of Engineering for Power, Trans. ASME. April: 171-181.

Nicholas, J. C., Gunter, E. J., and Allaire, P. E. 1976b. Effect of residual shaft bow on unbalance response and balancing of a single mass flexible rotor, Part 2: Balancing. Journal of Engineering for Power, Trans. ASME. April: 182-187.

Yu, J. J., and Craggs, A. 1995. Transfer matrix method for finite element models of a chain-like structure under harmonic excitations. Journal of Sound and Vibration 187:169-175. 

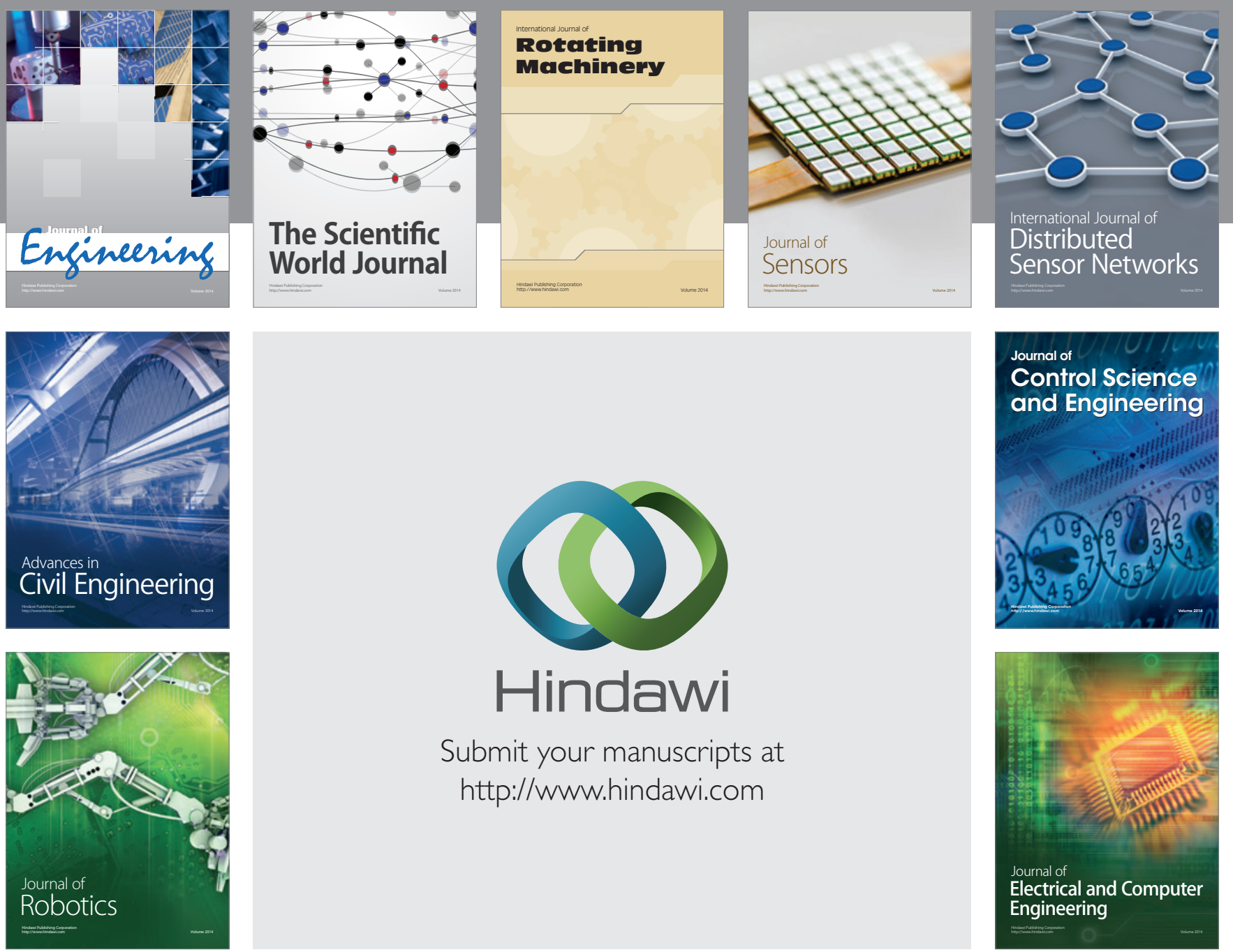

Submit your manuscripts at

http://www.hindawi.com
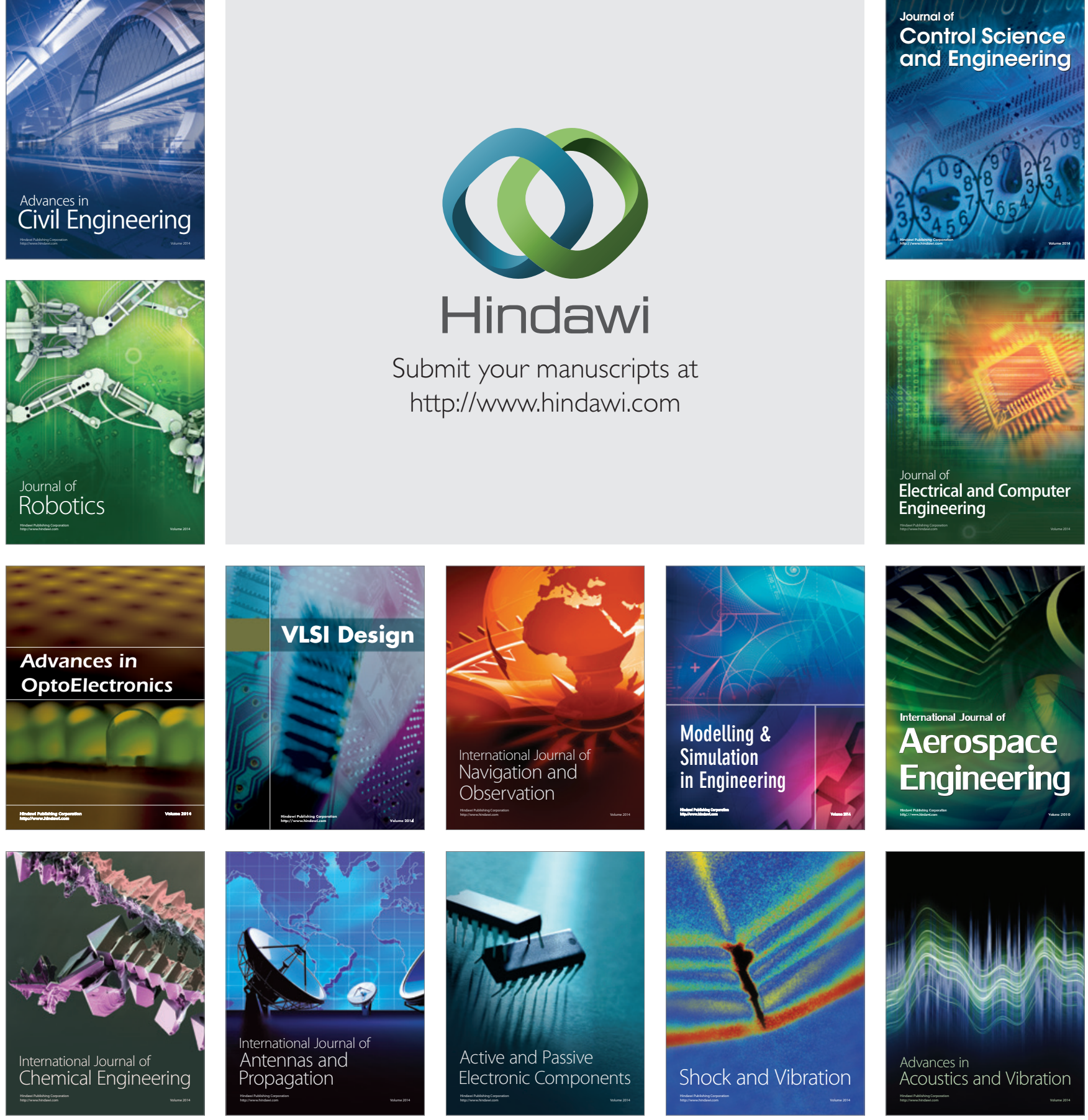